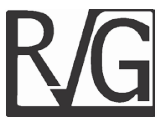

\title{
Franquicias del noreste mexicano: Incentivos de crecimiento*
}

\author{
Ayup González, Jannett** \\ Cavazos Arroyo, Judith***
}

\section{Resumen}

El desempeño de la franquicia suele abordarse desde la perspectiva de gestión comercial, sin embargo, la contribución interdisciplinar a la investigación sobre el desarrollo económico y sus implicaciones en la gestión han sido escasamente atendidos. México presenta una paradoja; mientras la comercialización de franquicias aumenta constantemente el número de marcas por la incursión de emprendedores, el total de estos establecimientos crece de manera inversa. Determinar los factores que favorecen la incursión de un emprendedor en la actividad franquiciadora y las expectativas de crecimiento, es el propósito de este estudio en la zona noreste del país por sus condiciones diferenciadas. La investigación es exploratoria y explicativa empleando como técnica de análisis un modelo de ecuaciones estructurales cuyos resultados sugieren factores de empresa y las características individuales como determinantes de las expectativas de nuevas unidades de negocio. Se evidencian empresarios jóvenes con perfil más emprendedor que inversor en franquicias, concluyendo que el tamaño de la franquicia y los incentivos empresariales definen la propensión al crecimiento por la apertura de nuevos establecimientos.

Palabras clave: franquicia; emprendimiento; empleo; crecimiento empresarial.

Recibido: 14-07-14 Aceptado: 29-07-15

Se agradece al Consejo Nacional de Ciencia y Tecnología de México por la financiación del proyecto TAMPS-2011-C35-185391, y a la Universidad Autónoma de Tamaulipas el apoyo dispensado.

** Doctora en Economía y Administración de Empresas, Universidad de Burgos/España. Profesora de marketing, Universidad Autónoma de Tamaulipas, México. Miembro del Sistema Nacional de Investigadores, México. Asesor especializado: Incubadora de Negocios, Universidad Autónoma de Tamaulipas, Consultora empresarial. E-mail: jannett.ayup@gmail.com, yanethay@uat.edu. $\mathrm{mx}$

*** Licenciada en Administración de Empresas, Maestra y Doctora en Dirección y Marketing. Profesora e investigadora en la Universidad Popular Autónoma del Estado de Puebla, México. Facilitadora de cursos en Bolivia, Brasil, Costa Rica, Estados Unidos, México y Nicaragua. E-mail: cavazosjudith01@gmail.com 


\title{
Incentives for growth in franchising in northwest Mexico
}

\begin{abstract}
The franchise performance is usually approached form the commercial management perspective; nevertheless the interdisciplinary contribution to economic development research and its implications in the management have been scarcely attended. Mexico presents a paradox: as the franchises commercialization is constantly incising the number of brands due to the entrepreneurs incursion, the total of these establishments is growing inversely. To determine the factors favoring the entrepreneur incursion in franchising activity and the growth expectations is the aim of this study in the north east region of the country due to its differentiated conditions. The research is exploratory and explicative using a structural equations model as analysis technique whose results suggest company features and the individual characteristics as determinants of the new business units expectations. Young businessmen with more of an entrepreneur profile than a franchises investor are evidenced concluding that the size of the franchise and the business incentives define the growing tendency by the opening of new establishments.
\end{abstract}

Key words: franchise; entrepreneurship; employ; business growth.

\section{Introducción}

La literatura de la Economía de la Empresa no suele enfocar la investigación en franquicia en relación al empleo, aunque reconoce su relevancia económica. Schumpeter (1934) explica que la renta y la gestión organizativa se encuentran vinculadas fuertemente al perfil del empresario. Para Shane et al (2006) la longevidad de las franquicias es ligeramente superior a otros tipos de negocio, pero ello difiere si se tiene en cuenta que la estructura de un sistema franquiciador es heterogénea y relacionada con los factores particulares de cada región (Acs y Audretsch, 1993). A ello se agrega la eficacia de las políticas públicas para incentivar la economía a través de iniciativas emprendedoras con mejor propensión a la supervivencia (Díaz et al, 2005). Entonces, podría decirse que el crecimiento económico, entendido como generador de empleo y renta, resulta del crecimiento empresarial, siendo crítica la longevidad de la firma para mantener su contribución económica.

Usualmente, se espera que una iniciativa empresarial bajo el formato de franquicia cuente con una gestión estandarizada, reconocimiento de marca, facilidad para comercializar y rápido crecimiento, sin embargo, esto no es igual si la plataforma para aumentar el número de establecimientos se compone en su mayoría por emprendedores o inversores de negocios. La visión y capacidades empresariales difiere, y con ello su contribución a la economía, cobrando importancia el perfil del emprendedor en franquicia que orienta sus esfuerzos al aumento constante de unidades de negocio. Así, la investigación muestra insuficiente evidencia empírica para relacionar las decisiones de franquicia y el empleo; en realidad se viene observando un contrasentido: mientras gran parte de los emprendedores incursionan en la franquicia como forma de autoempleo, los empresarios o inversores lo hacen como 
oportunidad de beneficios superiores. Díez de Castro et al (2008) distinguen y enfatizan el tamaño, crecimiento, sobrevivencia, intensidad del franquiciamiento e internacionalización en la gestión de franquicia, mientras que Revuelto-Taboada et al (2011) se interesan en el aumento de la participación en el mercado a través de estrategias en las unidades establecidas y el incremento de la creación de empleos en ellas.

La relevancia de la franquicia se exhibe por su naturaleza de expansión, dinamizando la economía de los países debido a las ventajas competitivas alcanzadas por sus prácticas comerciales (Welsh et al, 2006, Hoffman y Preble, 2003). En muchos países emergentes se ha utilizado como acelerador de su economía (Alon, 2006). Al respecto, Tormo y Asociados (2012) consultores en franquicia destaca a China con 4.000 marcas, seguido por Estados Unidos con 3.000; en Europa el país con mayor número de cadenas es Turquía con 1.843, mientras que en Iberoamérica el líder es Brasil con 2.031 marcas que producen 837.882 empleos, seguido por México con 1.013 redes, generando 750.000 empleos directos en 75.000 establecimientos.

La creación de empleo desde la franquicia es importante por su contribución a las economías locales al incrementar la base fiscal y promover la modernización económica. En Estados Unidos los negocios de franquicia tienen un promedio de treinta y tres puestos de trabajo -trece directos y veinte o más indirectos-, por unidad de negocio o establecimiento, México genera en promedio diez empleos directos por unidad de venta (Asociación Mexicana de Franquicias, 2014). En este sentido, es deseable emplear estrategias corporativas (Alon, 2004) y reconocer el papel de los mecanismos de apoyo locales para ampliar las oportunidades de expansión de las franquicias (Gillis y Castrogiovanni, 2012; Welsh et al, 2006) y la eficacia de los instrumentos de apoyo gubernamental sustentados en la Teoría Económica Institucional y Creación de Empresas (Díaz et al, 2005).

Para destacar la importancia de la franquicia en la economía mexicana, conviene mencionar que a pesar de que en 2012 ocupaba el décimo primer lugar, en importancia mundial por el número de franquicias -1.013 marcas-, España, contaba con 1.069 (Tormo y Asociados, 2012), casi el doble respecto de la extensión territorial de México-. Actualmente, se encuentra en etapa de experimentación del ciclo de vida en el ámbito internacional, y para acelerar su crecimiento y consolidación requiere de la creación de empresas y empleos mejor remunerados, por lo que al mostrar un menor riesgo en inversión el gobierno mexicano ha decidido apoyar el sector, especialmente para franquicias de baja inversión (Jacob, 2014). Varios estudios subrayan la potencialidad de crecimiento de las franquicias en México, destacando su tamaño y dinamismo (Welsh et al, 2006; Lafontaine y Oxley, 2004), ello constituye uno de los argumentos de justificación del presente estudio.

Para la Asociación Mexicana de Franquicias (2014), las franquicias activas son sólo 500 , de las cuales el $88 \%$ son marcas mexicanas; del total de redes, el $80.1 \%$ de las marcas cuenta con menos de 50 establecimientos y se reportan 500 mil empleos generados por esta actividad. Sin embargo, en la zona noreste de México, la última década ha sido afectada por factores macroeconómicos que han desincentivado la actividad emprendedora y empresarial, lo cual ha sido atenuado por la actividad franquiciadora impulsada con el apoyo del Plan Nacional de Franquicias iniciado en 2006. 
Al relacionar la franquicia en el ámbito del emprendimiento, la gestión empresarial y la economía, surge el interés de examinar si el futuro franquiciador $o$ franquiciado se apropia de las ventajas creadas por la franquicia para lograr el crecimiento y éxito empresarial; lo anterior se debe, bien al conocimiento adquirido -know how- (Audretsch y Thurik, 2001), o a su actuación empresarial, producto de su orientación personal a los negocios.

Siguiendo el planteamiento de Audretsch y Thurik (2001), asociado con los fundamentos microeconómicos del crecimiento y la literatura del desarrollo endógeno que enfatizan el rol del emprendimiento, se explora la visión e incentivos del emprendedor en su rol de empresario, y el tamaño y ventajas competitivas de la franquicia; relacionadas con las expectativas de apertura de nuevas unidades de negocio. Se pretende ofrecer un modesto marco referente para el desarrollo de modelos de crecimiento desde el sector de la franquicia como formato de negocio.

La presente es una investigación empírica de tipo exploratorio y explicativo. Para determinar la población objeto de estudio se emplearon los siguientes criterios: 1) ser una firma de franquicia identificada en el Catálogo Nacional de Franquicias del Instituto Nacional de Estadística, Geografía e Informática (INEGI, 2012) de México y, 2) contar con al menos 3 años de antigüedad en el mercado mexicano. Para discriminar si la firma corresponde al formato de franquicia fueron después INEGI: 1) el Directorio Oficial de Franquicias de la Asociación Mexicana de Franquicias A. C. y, 2) portal web de cada cadena.
Para la recolección de datos, con la intención de censar, se identificaron los establecimientos, recurriendo al método de muestreo polietápico por conveniencia en el centro, norte y sur del estado de Tamaulipas -el noreste mexicano- donde se concentran tres cuartas partes de la población urbanas más atractivas para el franquiciamiento. Se aplicó una encuesta personal a propietarios de la marca de franquicia o responsables de las unidades operativas en 189 establecimientos de 82 marcas, siendo descartadas 53 por inconsistencias en las respuestas o perfil del entrevistado y 23 no respondieron, obteniéndose 114 encuestas válidas.

Las 82 cadenas de franquicia fueron identificadas aleatoriamente de los 12 sectores más representativos de su actividad comercial en retail -comercio detallista- y servicios, de origen mexicano o extranjero, con al menos 3 años de haberse establecido en el área geográfica definida en el estudio. El instrumento consta de un apartado con características demográficas y 5 constructos: 1) visión, 2) incentivos de crecimiento, 3) tamaño de la franquicia, 4) ventajas competitivas y 5) expectativa de apertura de nuevos establecimientos. La encuesta fue validada mediante el método Fuzzy Delphi ${ }^{1}$ por 3 expertos académicos en emprendimiento, economía y franquicia, 4 representantes de gobierno e instituciones para el desarrollo económico en México y en el extranjero, 3 empresarios en franquicia y una consultora en franquicia. En el análisis estadístico se aplicaron modelos de ecuaciones estructurales, en específico la técnica de cuadrados mínimos parciales con el software Smart PLS versión 2.0.M3 (Ringle et al, 2005).

1 Para mejor comprensión del método consultar Ho y Wang (2008). 
La contribución del estudio se da en función de: 1) La identificación del tamaño del sistema franquiciador tamaulipeco, 2) la caracterización del perfil del franquiciador en su papel de emprendedor o inversor y 3) la determinación de la relación del perfil del franquiciador o franquiciado con sus proyecciones de crecimiento.

\section{Creación de empresas en la franquicia}

El modelo de la Teoría Económica Institucional y Creación de Empresas impacta en la creación de empleo, innovación y crecimiento, y desarrollo económico (Díaz et al, 2005), sin embargo, en el contexto de franquicia mexicano no se ha reconocido en ninguna de estas dimensiones. En el continente americano existe una intensa comercialización de cadenas franquiciadoras, que abren establecimientos tanto propios como concedidos a emprendedores atraídos por el éxito de este tipo de negocios. Siguiendo a Hoffman y Preble (2003), Barney (1991) y Porter (1982), las ventajas competitivas de estas firmas se apoyan en estrategias gerenciales y de marketing conformando barreras de entrada y salida en este sector de actividad.

Para las franquicias, Hoffman y Preble (2003) destacan como ventajas competitivas: a) economías de escala, b) activos intangibles -la imagen de marca o los procesos de operación-, c) experiencia del franquiciador $y \quad d)$ conocimiento del mercado; ventajas que explican la conversión de empresas independientes en franquicias. En tanto, Lillis et al (1976) compendian las ventajas competitivas de la franquicia en: 1) rápido acceso a mercados, 2) reducción de costo de capital, 3) distribución del riesgo en el canal de distribución y 4) propietarios-operadores altamente motivados; agregando los autores otras ventajas diferenciales como: 5) el reparto de trabajo de la gestión, 6) protección de acciones contra la confianza, 7) capacidad de brindar servicio en áreas geográficas distantes, 8) promoción de negocios franquiciados y 9) reducción de la concentración económica.

Bajo la premisa que las rentas de la franquicia se aproximan en un contexto macroeconómico al empleo generado, esta investigación propone tener en cuenta que el emprendedor en franquicia puede orientarsealautoempleooagenerarempleo y contribuir en mayor medida a la economía nacional. De las variables incorporadas, la variable latente expectativas de apertura de nuevos establecimientos recibe el efecto de los factores que representan las características individuales del emprendedor e inversor, mientras que las restantes refieren a atributos de la firma. El modelo explicativo resultante asume que la orientación del emprendedor en franquicia hacia el empleo es determinado por su visión de crecimiento, limitada en caso del autoempleo y amplia en caso de perfil de empleador; actuando en dos vertientes, con diferentes intensidad en su contribución económica: 1) como propietario de un negocio con uno o varios establecimientos o 2) como un franquiciador propietario de la marca o bien un inversor en franquicia que comercializa el concepto. Esta distinción viene de la literatura en emprendimiento asociando la franquicia como una forma de emprendimiento (Urbano y Toledano, 2008; Verheul et al, 2006; Reynolds et al, 2001).

Las características de los emprendedores y su actuación gerencial difieren por el estado en que el fundador inicia el negocio, sus motivaciones e incentivos, y el rol que desempeñan no deja de ser importante en las políticas económicas de los gobiernos 
(Caliendo y Kritikos, 2010). Al respecto, Schumpeter (1934) identifica tres tipos de emprendedores: 1) emprendedores en pequeños negocios, 2) intraemprendedores, es decir, aquellos con espíritu emprendedor en ideas y acciones dentro de una empresa y 3 ) los grandes emprendedores con mandos gerenciales o propietarios de empresas. Por su parte Wennekers y Thurik (1999) distinguen dos de los tres tipos de emprendedores bajo el enfoque schumpeteriano. Por tanto, la visión de un negocio se asocia en esta investigación al perfil del poseedor de una franquicia como emprendedor o inversionista. Entonces, el emprendedor pudiera tomar la decisión de ser propietario de uno o algunos establecimientos, o ser inversor esperando una participación financiera con el propósito principal de cosechar un retorno económico (Grünhagen y Mittelstaedt, 2005).

La visión del emprendedor sin aversión al riesgo, independientemente de su habilidad y capacidad para identificar oportunidades de negocio (Weenekers y Thurik, 1999), tendría la intención de desarrollar una franquicia, operar una franquicia máster ${ }^{2}$, o incursionar con varias unidades de venta o en distintas marcas de franquicia. Norton y Moore (2006) no encontraron diferencias entre emprendedores y no emprendedores respecto de los riesgos tomados, por ello, la primera hipótesis se plantea de tal forma:

Hipótesis 1: La visión del emprendedor define la expectativa de apertura de nuevos establecimientos.

Si un emprendedor decide incorporarse a una cadena de fran-quicias, su orientación al crecimiento pareciera natural, sin embargo, existe la posibilidad de que se adhiera a ella porque este tipo de negocios ofrecen incentivos de crecimiento relacionados con ingresos y rentabilidad. Tanto los programas de apoyo y asistencia de los gobiernos suelen ser incentivos positivos, mientras que los métodos de control que ejerce el franquiciador para asegurar la estandarización en las unidades de negocio distantes geográficamente serían incentivos negativos. Aquellos incentivos de crecimiento como la ampliación del negocio o la apertura de nuevos establecimientos están vinculados a la contratación de personal y la eficiencia productiva (Niefert, 2010; Ramírez et al, 2007; Sardy y Alon, 2007), incentivos que serán apreciados por el emprendedor según sus percepciones y aspiraciones. La segunda hipótesis supone que:

Hipótesis 2: Los incentivos de crecimiento se relacionan con las expectativas de apertura de nuevos establecimientos.

Este estudio espera que cuanto mayor sea la intención de hacer crecer su empresa, el emprendedor alcanzará mayor tamaño organizacional. Así lo sugiere el estudio de Caliendo y Kritikos (2010) al encontrar que el emprendedor es guiado por factores de motivación e incentivos en un push-pull al mismo tiempo, el tamaño del negocio creado por el emprendedor determinó las diferencias en el éxito y supervivencia del negocio y fue conceptualizado por las cantidades de inversión en capital, el número de empleados adicionales creados y el nivel de ingreso. Sardy y Alon (2007) también evidencian diferencias en el

2 Franquicia máster es el término empleado para el franquiciado que a nombre del franquiciador comercializa un concepto de franquicia en un área geográfica determinada formalizando y operando contratos de franquicia. 
tamaño de empresa para franquiciados y emprendedores nacientes ${ }^{3}$. Por tanto, la hipótesis relativa al tamaño de la franquicia es:

Hipótesis 3: Los negocios de franquiciadores $y$ franquiciados con mayor tamaño empresarial se relacionan positivamente con las expectativas de nuevos establecimientos.

En la empresa las ventajas competitivas crean barreras de entrada y salida en un sector de la actividad franquiciadora (Porter, 1985). Dichas estrategias y ventajas surgen de la visión y actuación del emprendedor en franquicia, sea cual fuere su rol -franquiciador o franquiciado-, pero dotados de distintos niveles de capacidades, habilidades y experiencia por lo que su contribución a la generación de puestos de trabajo será en ese sentido. Así, a partir de estos argumentos se plantea la hipótesis 4 :

Hipótesis 4: La conformación de ventajas competitivas en una iniciativa emprendedora de franquicia influye positivamente con la pretensión de abrir nuevos establecimientos.

La literatura de ventaja competitiva (Porter, 1985; Wernerfelt, 1984; Peteraf, 1993) surge de la Teoría de Crecimiento de la Firma (Penrose, 1962) y su aplicación en la franquicia, coincide con el concepto de ventaja competitiva ofrecido por Barney (1991) con su focalización a la firma. La gestión estratégica del franquiciado se centra en el desarrollo de alguna ventaja competitiva y de ello se favorecen los esfuerzos de comercialización y gestión de los recursos de franquicia. Aunque, las marcas de franquicia ostentan ventajas como penetración rápida y con bajo costo a nuevos mercados, éstas no son necesariamente ventajas competitivas ${ }^{4}$, para ello se requiere tiempo en el desarrollo.

Esta investigación organizó los indicadores de acuerdo al diamante de ventajas competitivas de Porter (1985,1990). Derivado de ello, el constructo teórico ventajas competitivas (VC) fue definido por las dimensiones: 1) factores condicionantes, 2) proveeduría y soporte, 3) estrategias comerciales y, 4) estrategias organizacionales. Respecto de ventajas competitivas con los fundamentos expuestos se proponen las siguientes hipótesis:

Hipótesis 5: Los factores condicionantes de la operación de una franquicia tienen una relación positiva con la ventaja competitiva.

Hipótesis 6: Los aspectos de proveeduría y soporte del franquiciador definen la ventaja competitiva de una franquicia.

Hipótesis 7: Las estrategias comerciales que despliegan los negocios de franquicia se relacionan positivamente con las ventajas competitivas de la marca.

Hipótesis 8: Las estrategias organizacionales que practica una franquicia definen su ventaja competitiva.

Los estudios de Audretsch y Thurik (2001) y Audretsch et al (2001) enfatizan la ambigüedad de la relación entre el emprendimiento y cambios económicos porque cambios en el desempleo tienen impacto positivo en el emprendimiento, pero también, cambios en el nivel de emprendimiento tienen influencia negativa en el desempleo, porque son dinámicas de relación inter-temporal y en dirección opuesta. Este trabajo pretende en un

3 Se conoce como emprendedor naciente al que compromete recursos en el desarrollo de una idea de negocio (Ver Global Entrepreneurship Monitor).

$4 \quad$ Para profundizar en las premisas propuestas por Barney consultar referencia de 1991. 
primer acercamiento al emprendimiento en franquicia; identificar las diferencias en el perfil del emprendedor y su articulación con su propia proyección de empleos. El
Cuadro 1 describe las variables incluidas en modelo que relaciona positivamente todas las variables para explicar esta ambigüedad.

\section{Cuadro 1}

Variables de estudio

\begin{tabular}{|c|c|c|c|}
\hline CONSTRUCTO & $\begin{array}{l}\text { VARIABLES RELEVANTES INCORPORADAS DE LA } \\
\text { LITERATURA }\end{array}$ & $\begin{array}{l}\text { DISCIPLINAS } \\
\text { RELEVANTES }\end{array}$ & $\begin{array}{l}\text { UNIDADES DE } \\
\text { OBSERVACIÓN }\end{array}$ \\
\hline $\begin{array}{l}\text { Visión (VI) } \\
\text { (Weaven y Frazer, 2007; } \\
\text { Weenekers y Thurik, } \\
\text { 1999). }\end{array}$ & $\begin{array}{l}\text { X1-Aversión o propensión al riesgo } \\
\text { X2-Internacionalización } \\
\text { X3-Visión emprendedora } \\
\text { X4-Acceso a Capital para expansión } \\
\text { X5-Acceso a Financiamiento }\end{array}$ & $\begin{array}{l}\text { Emprendimiento } \\
\text { Franquicia }\end{array}$ & Individuos \\
\hline $\begin{array}{l}\text { Incentivos de } \\
\text { crecimiento (IC) } \\
\text { (Niefert, 2010; Ramírez } \\
\text { et al, 2007; Sardy y Alon, } \\
2007\end{array}$ & $\begin{array}{l}\text { X6- Estimula la contratación de personal } \\
\text { X7- Eficiencia productiva } \\
\text { X8- Impulso de apertura de nuevos establecimientos }\end{array}$ & $\begin{array}{l}\text { Emprendimiento } \\
\text { Franquicia } \\
\text { Economía }\end{array}$ & $\begin{array}{l}\text { Individuos } \\
\text { Firmas } \\
\text { Industria }\end{array}$ \\
\hline $\begin{array}{l}\text { Tamaño (TA) } \\
\text { (Caliendo y Kritikos, 2010; } \\
\text { Sardy y Alon, 2007) }\end{array}$ & $\begin{array}{l}\text { X9- Núm. Establecimientos propios } \\
\text { X10- Núm. Establecimientos franquiciados } \\
\text { X11- Núm. Establecimientos en Tamaulipas }\end{array}$ & $\begin{array}{l}\text { Emprendimiento } \\
\text { Franquicia }\end{array}$ & $\begin{array}{l}\text { Individuos } \\
\text { Firmas } \\
\text { Industria }\end{array}$ \\
\hline $\begin{array}{l}\text { Ventajas competitivas } \\
\text { (VC) } \\
\text { I. Factores condicionantes } \\
\text { (FC) } \\
\text { (Pilling, 1991; Porter, 1990; } \\
\text { Porter, 1982) }\end{array}$ & $\begin{array}{l}\text { X13- Categoría de la franquicia } \\
\text { X14- Accesibilidad geográfica } \\
\text { X15- Cobertura de entrega } \\
\text { X16- Concentración de competidores } \\
\text { X17- Capital de inversión } \\
\text { X18-Instalaciones adecuadas }\end{array}$ & $\begin{array}{l}\text { Emprendimiento } \\
\text { Franquicia } \\
\text { Economía } \\
\text { Industrial }\end{array}$ & $\begin{array}{l}\text { Individuos } \\
\text { Firmas } \\
\text { Industria }\end{array}$ \\
\hline $\begin{array}{l}\text { Ventajas competitivas } \\
\text { (VC) } \\
\text { II. Proveeduría y soporte } \\
\text { (PS) } \\
\text { (Pilling, 1991; Porter, 1990; } \\
\text { Porter, 1982) }\end{array}$ & $\begin{array}{l}\text { X19- Ubicación de la empresa para abastecimiento } \\
\text { X20- Colaboración de los proveedores } \\
\text { X21- Entregas puntuales de los proveedores } \\
\text { X22- Calidad de los productos y servicios de } \\
\text { proveedores } \\
\text { X23-Plan de contingencia ante demandas inesperadas } \\
\text { X24-Planeación de las compras }\end{array}$ & $\begin{array}{l}\text { Emprendimiento } \\
\text { Franquicia } \\
\text { Economía } \\
\text { Industrial }\end{array}$ & $\begin{array}{l}\text { Individuos } \\
\text { Firmas } \\
\text { Industria }\end{array}$ \\
\hline $\begin{array}{l}\text { Ventajas competitivas } \\
\text { (VC) } \\
\text { III. Estrategias } \\
\text { Comerciales (EC) } \\
\text { (Pilling, 1991; Porter, 1990; } \\
\text { Porter, 1982) }\end{array}$ & $\begin{array}{l}\text { X25- Mercado objetivo definido } \\
\text { X26- Conocimiento del segmento } \\
\text { X27- Empleo de TICs } \\
\text { X28- Sistemas de calidad } \\
\text { X29- Orientación de recursos a un mercado con } \\
\text { demanda suficiente } \\
\text { X30-Planificación de estrategias comerciales }\end{array}$ & $\begin{array}{l}\text { Emprendimiento } \\
\text { Franquicia } \\
\text { Economía } \\
\text { Industrial }\end{array}$ & $\begin{array}{l}\text { Individuos } \\
\text { Firmas } \\
\text { Industria }\end{array}$ \\
\hline $\begin{array}{l}\text { Ventajas competitivas } \\
\text { (VC) } \\
\text { IV. Estrategias } \\
\text { organizacionales (EO) } \\
\text { (Pilling, 1991; Porter, 1990; } \\
\text { Porter, 1982) }\end{array}$ & $\begin{array}{l}\text { X31- Sistemas de control y gestión administrativa } \\
\text { X32- Capacidad de crear ventaja competitiva } \\
\text { X33- Sistemas de información para requerimientos } \\
\text { X34- Redes de cooperación } \\
\text { X35- Promoción de competencia interna }\end{array}$ & $\begin{array}{l}\text { Emprendimiento } \\
\text { Franquicia } \\
\text { Economía } \\
\text { Industrial }\end{array}$ & $\begin{array}{l}\text { Individuos } \\
\text { Firmas } \\
\text { Industria }\end{array}$ \\
\hline $\begin{array}{l}\text { Expectativas de apertura } \\
\text { de establecimientos (EA) } \\
\text { (Audretsch et al, 2001). }\end{array}$ & X12- Expectativas número de nuevos establecimientos & $\begin{array}{l}\text { Emprendimiento } \\
\text { Franquicia }\end{array}$ & $\begin{array}{l}\text { Individuos } \\
\text { Firmas }\end{array}$ \\
\hline
\end{tabular}

Fuente: elaboración propia. 
El modelo hipotético ${ }^{5}$ incorpora las 3 variables ( $\mathrm{VI}, \mathrm{IC}$ y $\mathrm{VC}$ ) que contribuyen a explicar la expectativa de nuevas unidades de negocio (EA) del franquiciado o franquiciador, siendo la visión una motivación, y como incentivos de crecimiento las variables incentivos de crecimiento, tamaño de la firma y ventajas competitivas. El análisis empírico del modelo de crecimiento esperado en la franquicia por los emprendedores se plantea de la siguiente forma para su análisis estadístico.

$$
\mathrm{EAi}=\mathrm{\alpha}_{1}[\mathrm{VI}]+\mathrm{\alpha}_{2}[\mathrm{ICi}]+\mathrm{\alpha}_{3}[\mathrm{TAi}]+\mathrm{\alpha}_{4}[\mathrm{VCi}]
$$

(Ecuación 1)

Donde la i representa la marca de franquicia, [EA] es la variable independiente expectativa del crecimiento en número de nuevos establecimientos, [VI] es la variable que recoge la visión del emprendedor, [TA] es el número de establecimientos de la franquicia en el estado de Tamaulipas, [VC] son las ventajas competitivas que afirma poseer la franquicia entrevistada y $e$ es el término de error. Resumiendo, se tiene un modelo hipotético para explicar el crecimiento de la franquicia dado el perfil del emprendedor, ya sea orientación emprendedora u orientación al negocio ${ }^{6}$.

\subsection{Incentivos o motivación del emprendedor al crecimiento}

Antes de la presentación de los hallazgos se presenta la Tabla 1 para detallar el perfil de los entrevistados. En esta sección se analiza tanto la metodología empleada, como las variables y sus indicadores, la muestra poblacional, la evaluación de los efectos del modelo y el modelo final de las de expectativa de apertura de nuevas unidades de negocio de franquicia.

\section{Tabla 1}

Características demográficas de los entrevistados

\begin{tabular}{|c|c|c|c|c|c|}
\hline $\begin{array}{l}\text { Actividad de la } \\
\text { franquicia } \\
(n=110)\end{array}$ & $\%$ & $\begin{array}{l}\text { Estado Civil } \\
\qquad(n=112)\end{array}$ & $\%$ & $\begin{array}{l}\text { Nivel de estudios } \\
\quad(n=112)\end{array}$ & $\%$ \\
\hline Franquiciador & 2.7 & Soltero(a) & 33.6 & Secundaria incompleta & 0.9 \\
\hline Franquiciado & 10 & Casado (a) & 53.1 & Secundaria completa & 2.7 \\
\hline \multirow[t]{3}{*}{ Gerente/responsable * } & 87.3 & Unión libre & 9.7 & Preparatoria incompleta & 2.7 \\
\hline & & Divorciado (a) & 1.8 & Preparatoria completa & 23.2 \\
\hline & & Viudo (a) & 0.9 & Técnico incompleto & 3.6 \\
\hline Sexo $(n=112)$ & $\%$ & Perfil (n=98) & $\%$ & Licenciatura incompleta & 17.9 \\
\hline Masculino & 61.1 & Emprendedor & 87 & Licenciatura completa & 45.5 \\
\hline Femenino & 38.1 & Inversor & 13 & Maestría & 3.6 \\
\hline
\end{tabular}

*Nota: no se tuvo acceso a concesionarios de marca (multifranquiciantes) como forma de participación en la franquicia (alternativa 3 de respuesta).

Fuente: elaboración propia.

5 Para visualización se puede consultar la Figura 1 de este documento.

6 Para profundizar en la orientación emprendedora y la orientación al negocio, consultar Runyan et al (2008). 
El cuestionario incorporó las variables medidas del Cuadro 1 utilizándose escalas Likert para medir los constructos VI, IC, TA, EA y dicotómicas para los constructos FC, PS, EC, EO. Los indicadores fueron respaldados con las siguientes fuentes: $\mathrm{VI}$ (Tuunanen y Hyrsky, 2001), IC (Niefert, 2010; Sardy y Alon, 2007; Ramírez et al, 2007), TA (Niefert, 2010; Ramírez et al, 2007; Sardy y Alon, 2007), VC (Saavedra, 2012; Porter, 1990; Porter, 1982). Basado en el instrumento empleado por Saavedra (2012) para determinar la competitividad de la empresa, se estableció una agrupación de indicadores de acuerdo al diamante de ventajas competitivas de Porter (1990): FC, PS, EC y EO. La naturaleza de los constructos, fue verificada a través de la lista de criterios y características propuesta por Fassott (2006); cuatro constructos fueron modelados reflectivos (VI, IC, TA y EA). VC fue definido como constructo de segundo orden formativo, empleando la aproximación de indicadores repetidos (Wetzels et al, 2009), conformado por FC, PS, EC y EO (Wennekers y Thurik, 1999).

- Perfil de los entrevistados (PEI): la mayoría de los encuestados espera contar entre 1 y 6 establecimientos de la misma cadena de franquicia durante los próximos 3 años. Además, más de la mitad de los encuestados decidió dedicarse al negocio por necesidad (motivos de empleo o término relación laboral previa); el $87 \%$ reportó un perfil emprendedor relacionado con los motivos para iniciar un negocio de franquicia, capital disponible y sus expectativas de apertura de nuevas unidades de negocio.

Los resultados muestran que no existe traslape de ítems y ninguna de las correlaciones bivariadas entre los ítems es mayor a 0.90 (Hair et al, 2006), por lo que se asume que cada variable es lo suficientemente distinta para medir diferentes variables en el mismo constructo (Sekaran, 2002).Todos los constructos reflectivos endógenos, que poseen más de un indicador en el modelo teórico, presentan variables con factor de inflación de la varianza (FIV) menores a 10; indicando que no existen problemas de colinealidad (Henseler et al, 2008).

- Modelos de medición reflectivos: en los constructos con indicadores reflectivos (constructos latentes), las cargas examinadas se interpretan en la misma forma que las cargas en un análisis de componentes principales (Duarte y Raposo, 2010) y el criterio utilizado para evaluar la fiabilidad del indicador, es el tamaño (Seidel y Back, 2009). En constructos reflectivos con una sola variable, la carga es constante de 1 , asumiendo que no existe error en éstos; dado que la significancia estadística asume que existe cierto error o cierta variabilidad en la carga, el software Smart PLS no provee la significancia en tal caso (la carga no es evaluada). En base a la regla propuesta por Hulland (1999) de conservar los ítems con cargas de 0.7 o más, no todos los indicadores de los modelos de medición reflectivos, alcanzaron el nivel aceptable de fiabilidad y por tanto fueron eliminados. Sin embargo, como se aprecia en la Tabla 2, indicadores débiles (cargas mayores a 0.40) fueron retenidos por contribuir con la validez de contenido del modelo (Hair et al, 2011). 


\section{Tabla 2}

\section{Cargas de los indicadores reflectivos con más de un indicador}

\begin{tabular}{cccccccc}
\multicolumn{7}{c}{ (n=114) } \\
\hline \multirow{2}{*}{ Constructo } & Variable & \multicolumn{2}{c}{ Modelo 1 } & \multicolumn{2}{c}{ Modelo 2 } & \multicolumn{2}{c}{ Modelo 3 } \\
\cline { 2 - 8 } & & Cargas & Valor t & Cargas & Valor t & Cargas & Valor t \\
\hline \multirow{2}{*}{ Visión (VI) } & X1 & 0.220 & 0.721 & - & - & - & - \\
& X2 & 0.296 & 1.140 & 0.304 & 1.170 & - & - \\
& X4 & $0.752^{*}$ & 4.533 & $0.753^{*}$ & 6.835 & $0.751^{*}$ & 6.059 \\
& X5 & $0.828^{*}$ & 5.454 & $0.828^{*}$ & 8.078 & $0.830^{*}$ & 8.719 \\
Incentivos de & X6 & $0.802^{*}$ & 4.356 & $0.685^{*}$ & 5.143 & $0.688^{*}$ & 4.866 \\
crecimiento & X7 & $0.539^{*}$ & 2.927 & $0.539^{*}$ & 3.261 & $0.5839^{*}$ & 2.915 \\
(IC) & X8 & $0.766^{*}$ & 7.564 & $0.766^{*}$ & 7.475 & $0.766^{*}$ & 7.699 \\
\hline \multirow{2}{*}{\begin{tabular}{c} 
Tamaño (TA) \\
\cline { 3 - 9 }
\end{tabular}} & X9 & 0.358 & 1.396 & 0.267 & 1.188 & - & - \\
& X10 & 0.309 & 1.192 & - & - & - & - \\
\hline
\end{tabular}

* Significativo con alpha de 0.01 el valor t es mayor a 2.57 para una prueba de dos colas.

Fuente: elaboración propia.

Para los constructos reflectivos con más de un indicador, la validez de convergencia es evaluada con el valor del Promedio de Varianza Extraída (Average Variance Extracted, AVE). En el modelo final (modelo 3), los constructos reflectivos $\mathrm{VI}$ e IC, presentan un valor por arriba del límite del Criterio de Fornell y Larcker (1981) $(\mathrm{min}=0.507)$ (resultados no mostrados). En la Tabla 3 se muestran las cargas cruzadas de los indicadores reflectivos; todos ellos muestran una carga mayor en valor absoluto en el constructo al que han sido asignados, en relación a cualquier otro constructo reflectivo (Seidel y Back, 2009).

El coeficiente Rho de DillonGoldsteins evalúa la consistencia interna (Fornell y Larcker, 1981) para los constructos reflectivos $\mathrm{VI}$ e IC, fue mayor a 0.750 (resultados no mostrados), excediendo el valor mínimo aceptable de
0.70 (Seidel y Back, 2009). En cuanto a la validez discriminante. El promedio de varianza extraída para los constructos reflectivos es mayor a 0.507 , por arriba del valor aceptable de acuerdo a Fornell y Larcker (1981). Tal validez para los modelos de medición reflectivos también es demostrada cuando la raíz cuadrada del promedio de varianza extraída de cada constructo es mayor que la correlación con cualquier otra variable latente (Seidel y Back, 2009). En la Tabla 4 se compara la raíz cuadrada de AVE en la diagonal con el triángulo inferior de la matriz que contiene las correlaciones entre constructos. En el modelo, no se revelan problemas de validez discriminante, todos los indicadores muestran cargas mayores en su constructo con respecto a otros reflectivos (Duarte y Raposo, 2010). 
Tabla 3

Cargas cruzadas de los indicadores reflectivos y formativos en el modelo $3(n=114)$

\begin{tabular}{|c|c|c|c|c|c|c|c|c|}
\hline \multicolumn{9}{|c|}{ Constructo } \\
\hline Ítem & VI & IC & TA & PEI & FC & PS & EC & EO \\
\hline $\mathrm{x} 3$ & 0.591 & 0.218 & 0.201 & -0.095 & 0.136 & 0.133 & 0.197 & 0.171 \\
\hline $\mathrm{X} 4$ & 0.858 & 0.309 & 0.216 & -0.187 & 0.153 & 0.166 & 0.211 & 0.177 \\
\hline$\times 5$ & 0.796 & 0.299 & 0.137 & -0.174 & -0.064 & -0.031 & -0.049 & -0.075 \\
\hline $\mathrm{X} 6$ & 0.275 & 0.703 & 0.144 & -0.063 & -0.003 & 0.018 & 0.056 & 0.084 \\
\hline $\mathrm{X} 7$ & 0.324 & 0.909 & 0.056 & -0.108 & 0.062 & 0.174 & 0.210 & 0.174 \\
\hline X11 & 0.235 & 0.106 & 1.000 & -0.684 & 0.075 & -0.028 & 0.007 & 0.039 \\
\hline X12 & -0.143 & -0.059 & -0.737 & 0.953 & -0.074 & 0.077 & 0.052 & 0.031 \\
\hline X13 & -0.278 & -0.184 & -0.297 & 0.718 & -0.091 & -0.033 & -0.016 & 0.003 \\
\hline X15 & -0.243 & -0.306 & -0.128 & 0.082 & -0.113 & -0.053 & -0.129 & -0.061 \\
\hline X16 & -0.077 & -0.048 & 0.138 & -0.090 & 0.445 & 0.353 & 0.337 & 0.342 \\
\hline X17 & 0.013 & 0.010 & 0.028 & 0.015 & 0.377 & 0.289 & 0.275 & 0.260 \\
\hline X18 & 0.164 & 0.087 & 0.050 & -0.061 & 0.454 & 0.351 & 0.322 & 0.315 \\
\hline X19 & 0.148 & 0.107 & -0.041 & -0.138 & 0.584 & 0.488 & 0.392 & 0.382 \\
\hline $\mathrm{X} 20$ & -0.006 & -0.039 & 0.009 & 0.014 & 0.710 & 0.516 & 0.549 & 0.516 \\
\hline X21 & 0.068 & 0.129 & 0.033 & 0.046 & 0.549 & 0.729 & 0.566 & 0.569 \\
\hline $\mathrm{X} 22$ & 0.011 & 0.039 & 0.024 & -0.062 & 0.527 & 0.742 & 0.631 & 0.592 \\
\hline $\mathrm{X} 23$ & 0.019 & 0.060 & 0.132 & -0.153 & 0.458 & 0.507 & 0.387 & 0.372 \\
\hline X24 & -0.070 & -0.026 & -0.155 & 0.175 & 0.410 & 0.462 & 0.422 & 0.327 \\
\hline X25 & 0.156 & 0.181 & -0.006 & 0.033 & 0.599 & 0.828 & 0.691 & 0.697 \\
\hline X26 & 0.192 & 0.143 & -0.072 & 0.096 & 0.554 & 0.698 & 0.559 & 0.546 \\
\hline X27 & -0.018 & 0.143 & -0.010 & 0.028 & 0.532 & 0.538 & 0.640 & 0.574 \\
\hline X28 & 0.173 & 0.032 & 0.126 & -0.120 & 0.522 & 0.542 & 0.656 & 0.580 \\
\hline X29 & 0.143 & 0.137 & 0.020 & 0.073 & 0.493 & 0.558 & 0.709 & 0.619 \\
\hline X30 & -0.024 & 0.088 & -0.109 & 0.181 & 0.505 & 0.490 & 0.609 & 0.534 \\
\hline X31 & 0.106 & 0.101 & 0.026 & 0.054 & 0.491 & 0.652 & 0.769 & 0.702 \\
\hline X32 & 0.189 & 0.246 & -0.038 & -0.026 & 0.515 & 0.609 & 0.727 & 0.648 \\
\hline X33 & 0.116 & 0.206 & 0.101 & 0.002 & 0.445 & 0.488 & 0.518 & 0.587 \\
\hline X34 & -0.011 & 0.053 & 0.022 & 0.012 & 0.406 & 0.465 & 0.543 & 0.592 \\
\hline X35 & 0.058 & 0.069 & 0.010 & 0.023 & 0.529 & 0.621 & 0.693 & 0.777 \\
\hline X36 & 0.140 & 0.147 & 0.005 & -0.043 & 0.410 & 0.497 & 0.570 & 0.636 \\
\hline X37 & 0.055 & 0.107 & -0.010 & 0.079 & 0.537 & 0.524 & 0.599 & 0.684 \\
\hline
\end{tabular}

Fuente: elaboración propia. 


\section{Tabla 4}

\section{Matriz de correlaciones entre constructos y raíz de AVE mayor a} las correlaciones del modelo $3(n=114)$

\begin{tabular}{c|cccccccc}
\hline & EA & EO & EC & FC & PS & TA & VC & VI \\
\hline EA & $\mathbf{1}$ & & & & & & & \\
EO & 0.1280 & na & & & & & & \\
EC & 0.1562 & 0.8919 & na & & & & & \\
FC & 0.1301 & 0.7087 & 0.7439 & na & & & & \\
PS & 0.0976 & 0.7932 & 0.8281 & 0.7641 & na & & & \\
TA & 0.4866 & 0.0392 & 0.0093 & 0.0805 & 0.0256 & na & & \\
VC & 0.1488 & 0.9280 & 0.9553 & 0.8417 & 0.9290 & 0.0256 & na & \\
VI & 0.2197 & 0.1399 & 0.1796 & 0.1251 & 0.1332 & 0.2482 & 0.1716 & na \\
\hline
\end{tabular}

na $=$ no aplica

Fuente: elaboración propia.

- Modelos de medición formativos: la magnitud y significancia de los pesos en los indicadores muestran la importancia de la contribución de la variable latente asociada (Duarte y Raposo, 2010) e interpretadas como un coeficiente de validez (Bollen, 1989). Diamantopoulus y Winklhofer (2001) recomiendan que indicadores no significativos no deberían ser considerados mediciones válidas del constructo relevancia respectivamente; el ítem 35 (peso=0.399) expone mayor trascendencia en formar EO (Tabla 5 ); otros sugieren que aquellos que contribuyan a la validez de contenido del constructo deben ser retenidos (Petter et al, 2007). Aun cuando los resultados de 500 submuestras bootstrap evidencien que el ítem 13 presenta un peso negativo no significativo, todos los ítems son retenidos en el modelo para su valoración (Chin, 1998), sin embargo, una relación sustancial debe ser verificada (Seidel y Back, 2009). Las variables más importantes son: para FC el ítem 20 (peso=0.566); el ítem 25 para PS (peso $=0.388$ ) y para EC, el ítem 31 (peso=0.296) y el ítem 35 (peso $=0.399$ ) para EO.

- Modelo estructural: este modelo explica el $30.20 \%$ de la varianza de EA $\left(R^{2}=0.302\right)$, directamente por $\mathrm{VI}, \mathrm{IC}, \mathrm{TA}$ y VC e indirectamente por FC, PS, EC y EO. Un boostrap con 500 submuestras fue empleado para estimar la significancia de los coeficientes path en el modelo y comparar los estimadores estadísticamente. Los resultados respaldan el $75 \%$ de las hipótesis (Tabla 6). Es decir, 5 de 8 paths principales son mayores a 0.2 , el estándar mínimo de significancia (Chin, 1998). El $75 \%$ fueron significativos en el sentido propuesto, con al menos una confianza del $95 \%$. No se apoyaron las hipótesis $\mathrm{H} 1$ y H4. 


\section{Tabla 5}

\section{Pesos de indicadores formativos en el modelo $3(n=114)$}

\begin{tabular}{|c|c|c|c|}
\hline Constructo & Variable & Pesos & Valor $\mathrm{t}$ \\
\hline \multirow{6}{*}{ Factores condicionantes (FC) } & X15 & $-0,073$ & 0,905 \\
\hline & $\mathrm{X} 16$ & $0.371^{*}$ & 5,211 \\
\hline & $\mathrm{X} 17$ & $0.275^{\star}$ & 3,435 \\
\hline & $\mathrm{X} 18$ & $0.293^{*}$ & 4,241 \\
\hline & $\mathrm{X} 19$ & $0.322^{*}$ & 4,558 \\
\hline & $\mathrm{X} 20$ & $0.566^{*}$ & 7,179 \\
\hline \multirow{6}{*}{ Proveeduría y soporte (PS) } & $\mathrm{X} 21$ & $0.174^{*}$ & 3,076 \\
\hline & $\mathrm{X} 22$ & $0.277^{*}$ & 5,038 \\
\hline & $\mathrm{x} 23$ & $0.128^{*}$ & 2,872 \\
\hline & $\mathrm{X} 24$ & $0.238^{*}$ & 4,578 \\
\hline & $\times 25$ & $0.388^{*}$ & 6,76 \\
\hline & $\mathrm{X} 26$ & $0.246^{*}$ & 5,011 \\
\hline \multirow{6}{*}{ Estrategias comerciales (EC) } & $\mathrm{X} 27$ & $0.263^{*}$ & 6,975 \\
\hline & $\mathrm{X} 28$ & $0.250^{*}$ & 6,723 \\
\hline & $\mathrm{X} 29$ & $0.158^{*}$ & 3,078 \\
\hline & $\mathrm{X} 30$ & $0.207^{*}$ & 5,149 \\
\hline & $\mathrm{X} 31$ & $0.296^{*}$ & 6,378 \\
\hline & X32 & $0.277^{*}$ & 6,368 \\
\hline \multirow{5}{*}{ Estrategias organizacionales (EO) } & $\mathrm{X} 33$ & $0.297^{*}$ & 6,633 \\
\hline & $\mathrm{X} 34$ & $0.292^{*}$ & 5,889 \\
\hline & X35 & $0.399^{*}$ & 6,401 \\
\hline & X36 & $0.233^{*}$ & 4,269 \\
\hline & $\times 37$ & $0.285^{*}$ & 5,768 \\
\hline
\end{tabular}

* Significativo con alpha de 0.01 el valor t es mayor a 2.57 para una prueba de dos colas.

Nota: dados los parámetros estadísticos establecidos, las variables x13 y x14 fueron eliminadas por no contribuir al modelo robusto.

Fuente: elaboración propia.

Con la aproximación de indicadores repetidos, todas las facetas de la dimensión Ventaja Competitiva (FC, PS, EC y EO) tienen una influencia significativa y positiva en esta dimensión de segundo orden (Tabla 6, valores $\mathrm{t}>1.96$ ), apoyando las hipótesis $\mathrm{H} 2, \mathrm{H} 3, \mathrm{H} 5, \mathrm{H} 6, \mathrm{H} 7$ y $\mathrm{H} 8$. Al respecto, PS y EC muestran la mayor contribución positiva en VC (pesos=0.319 y 0.347 respectivamente), comparado con el resto. No se encontró un efecto directo significativo de Ventaja Competitiva en Expectativa de apertura de nuevas unidades de negocio (valor $\mathrm{t}=0.959$ ), por lo que la influencia de Ventaja Competitiva (VC) de primer orden en la Expectativa de apertura (EA) (efectos indirectos), no ha podido ser evaluada. 
Franquicias del noreste mexicano: Incentivos de crecimiento Ayup González, Jannett y Cavazos Arroyo, Judith

\section{Tabla 6}

\section{Evaluación de los efectos en el modelo $3(n=114)$}

\begin{tabular}{cllccc}
\hline Hipótesis & & Path & $\begin{array}{c}\text { Coeficiente } \\
\text { path }\end{array}$ & Valor $\mathbf{t}^{*}$ & $\begin{array}{c}\text { Cumplimiento de } \\
\text { hipótesis }\end{array}$ \\
\hline H1 & VI & EA (+) & $-0,024$ & 0,3545 & Rechazo \\
H2 & IC & EA $(+)$ & $0,235^{*}$ & 3,3826 & No rechazo \\
H3 & TA & EA (+) & $0,452^{*}$ & 4,9342 & No rechazo \\
H4 & VC & EA (+) & 0,086 & 0,9593 & Rechazo \\
H5 & FC & VC $(+)$ & $0,162^{*}$ & 6,2087 & No rechazo \\
H6 & PS & VC (+) & 0,319 & 13,1711 & No rechazo \\
H7 & EC & VC (+) & $0,347^{*}$ & 14,3931 & No rechazo \\
H8 & EO & VC $(+)$ & $0,250^{*}$ & 10,4223 & No rechazo \\
\hline
\end{tabular}

* Significativo con alpha de 0.01 el valor t es mayor a 2.57, para una prueba de dos colas, Ho: el coeficiente path $=0$.

Fuente: elaboración propia.

Figura 1

Modelo final de expectativa de apertura de nuevos establecimiento de franquicia

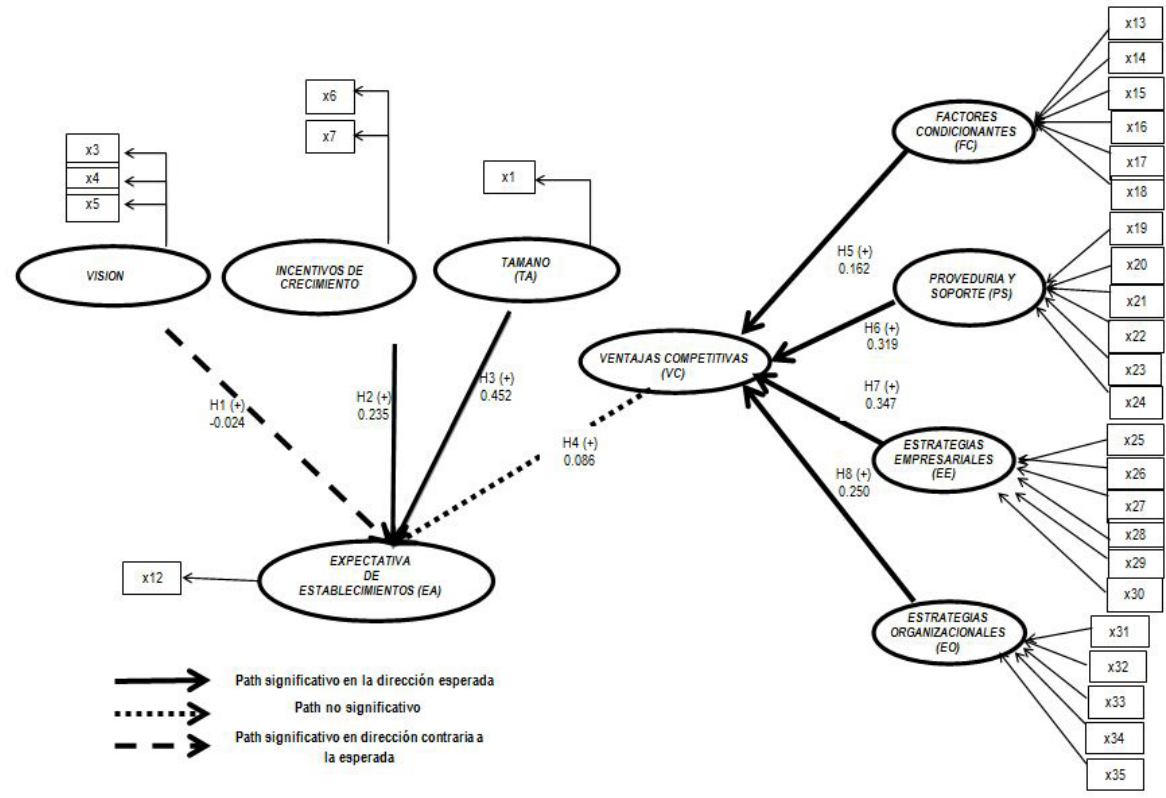

Fuente: elaboración propia. 
En el modelo obtenido con suficiente poder explicativo se observa la débil importancia que le otorgan franquiciadores y franquiciados a las ventajas competitivas creadas desde la gestión organizativa. La visión dada por la actitud emprendedora resultó no favorable para abrir nuevos establecimientos, quizá apoyándose en el formato de negocio probado y estandarizado que le facilitaría obtener ventajas competitivas forma rápida para la obtención de rendimientos económicos. Los hallazgos principales distinguen la mayor propensión a abrir nuevos establecimientos por aquellos franquiciados y franquiciadores con más de un establecimiento, que cuentan con cierta eficiencia productiva, ligados a firmas de gran tamaño o reconocimiento y alineados a incentivos contractuales. Esto supone un aliciente para profesionalizar la gestión de nuevos negocios de franquicia en búsqueda de mayor contribución a la economía de los países.

Los hallazgos destacables para la gestión y la estrategia son: 1) tanto emprendedores, empresarios jóvenes e inversores experimentados contribuyen al empleo en distinta intensidad de acuerdo a su tamaño y longevidad, 2) la franquicia en el noreste de México presentó en 2013 condiciones favorables, 3) los pequeños y medianos negocios de franquicia, descansan su falta de profesionalización en las ventajas competitivas de la franquicia en cuyo formato se apoyan para invertir con mayor certeza pero sin grandes aspiraciones de crecimiento y 4) la escasa presencia de inversores en franquicia que incursionaron en ella al detectar oportunidad de negocio.

La antigüedad de 27 años en el mercado y 8 en la franquicia ha permitido a estos negocios adquirir ciertas ventajas competitivas, no obstante, contrariamente a lo esperado, estas ventajas no han sido suficientes para promover crecimiento para los próximos 3 años. Una probable explicación implica que los emprendedores y empresarios jóvenes son los que impulsan la creación de franquicias, contribuyendo con el autoempleo, pero como no pretenden crecer, no generan empleos. Esto probablemente, es debido a la falta de disciplina administrativa, gestión estratégica y fortalezas incipientes en los emprendedores que distinguen oportunidad de negocio y no logran conformarse como multifranquiciados o multifranquiciadores, quienes con visión de largo plazo, poder de gestión en firmas competentes podrían estimular el crecimiento económico dada la experiencia que les confiere su supervivencia y mayor contribución a la economía por la longevidad de la firma.

\section{Conclusiones}

La relación del perfil de emprendedor en el noreste de México, asociado a su futuro desempeño gerencial está orientado a la proyección de empleo. Sus condiciones de crecimiento se vieron limitadas por factores macroeconómicos en la década previa y durante el estudio. Con preponderancia de emprendedores por sobre inversores en franquicia con fuerte influencia de Estados Unidos, país líder en franquicia. De igual forma se evidenció que: el tamaño de la franquicia, el operar más de un establecimiento, así como los incentivos de eficiencia productiva y gestión empresarial impactan positivamente la expectativa de apertura de establecimientos; aunque restringe de manera negativa la visión 
del emprendedor; presumiendo que la decisión de adoptar el formato de franquicia es tomada para enmendar debilidades de gestión organizativa y comercial al reconocerse que ofrece mayor garantía de sobrevivencia y retorno de inversión.

Dicho crecimiento restringido parece obedecer a factores externos que favorecieron las ventas y el tamaño de la organización para sobrevivir frente a la incertidumbre en el mercado y la inestabilidad política, económica y social. Los franquiciados y franquiciadores se apoyaron en el reconocimiento de marca por el tamaño de la cadena y la eficiencia productiva derivada para hacer frente a los competidores y mantenerse en el mercado. Se vieron atraídos por este formato de negocio para dominar el mercado a menor costo y tiempo y con mayor probabilidad de éxito.

Es deseable tener en cuenta que algunos franquiciados con un solo establecimiento no siempre logran transitar a multifranquiciante debido a dos razones fundamentales: el efecto de las características personales de cada individuo en la elección de la estrategia de gobierno y las causas por las que se integran a una cadena, pueden ser la limitada ambición de crecimiento y ver la franquicia como una forma cómoda de obtener ingresos.

Así, el modelo obtenido para proyectar el crecimiento de puestos de trabajo por la gestión acertada de una franquicia propone integrar en una futura línea de investigación, con fundamento en la Teoría Económica Institucional y creación de empresas, las acciones institucionales, de empresa y gobierno que de ser eficaces impulsarían aquellas empresas en dos vertientes: 1) emprendedores que sostienen el autoempleo y 2) inversores con resultados económicos superiores por su tamaño y sostenibilidad que comprometen cuantías financieras superiores y contratan un gran número de empleados apostando a mayores ingresos.

\section{Referencias bibiliográficas}

Acs, Zoltan y Audretsch, David (1993), Small Firms and Entrepreneurship: An East-West Perspective, Cambridge University Press, Cambdrige.

Alon, Ilan (2004), Global Franchising and Development in Emerging and Transitioning Markets, Journal of Macromarketing, Vol. 24, $\mathrm{N}^{\circ}$. 2, Editorial SAGE Publications, pp 156167.

Alon, Ilan (2006), Key success factors of franchising system in the retailing sector, SCMS Journal of Indian Management, Vol. 3, $\mathrm{N}^{\circ} 1$, Editorial SCMS Group, pp 29-36.

Asociación Mexicana de Franquicias. Boletines informativos (20022014), México Disponible en: www. franquiciasdemexico.org Fecha de consulta: 12 de Marzo de 2014.

Audretsch, David y Thurik, Roy (2001), What's new about the New Economy?, Sources of Growth in the Managed and Entrepreneurial Economies. Industrial and Corporate Change, No. 10, Editorial Oxford Journals, pp 267-315. Disponible en: http:// citeseerx.ist.psu.edu/viewdoc/d?doi $=10 \cdot 1 \cdot 1 \cdot 462 \cdot 1460 \&$ rep $=$ rep $1 \&$ type $=p$ df Fecha de consulta: 29 de julio de 2015.

Audretsch, David; Carree, Martin y Thurik, Roy (2001), Does Entrepreneurship reduce unemployment?. TI 074-3. Tinbergen Institute, Rotterdam and Amsterdam. Disponible en: http://www.econstor. eu/bits0419/85927/1/01074.pdf Fecha de consulta: 17 de Agosto de 2011.

Barney, Jay (1991), Firm Resources and Sustained Competitive Advantage, 
Journal of Management, Vol. 17, $\mathrm{N}^{\circ}$ 1, Editorial SAGE Publications, pp 99120.

Bollen, Kenneth (1989), Structural Equations with Latent Variables. (Primera Edición), Malden, MA: WileyInterscience.

Caliendo, Marco y Kritikos, Alexander (2010), Start-Ups by the Unemployed: Characteristics, Survival and Direct Employment Effects, Small Business Economics, Vol. $35, \mathrm{~N}^{\circ} 1$, Editorial Springer, pp 71-92.

Chin, Wynne (1998), The Partial Least Squares Approach to Structural Equation Modeling. Hoyle, R.H. (Editores MIS Quarterly). Disponible en: http://www.jstor.org/r/10.2307/ 249674 ? uid $=3738664$ \&uid $=2$ \&uid $=$ $4 \&$ sid=21104114892993. Fecha de consulta: 9 de marzo de 2002.

Diamantopoulus, Adamantios y Winklhofer, Heidi (2001), Index Construction with formative indicators: An alternative to scale development, Journal of Marketing Research, Vol. 38, $\mathrm{N}^{\circ}$ 2, Editorial American Marketing Association Journals, pp 269-27.

Díaz, Juan Carlos, Urbano, David y Hernández, Ricardo (2005), Teoría económica institucional y creación de empresas, Investigaciones Europeas de Dirección y Economía de la Empresa, Vol. 11, $\mathrm{N}^{\circ} 3$, Academia Europea de Dirección y Economía de la Empresa (AEDEM virtual), pp 209-230.

Díez de Castro, Enrique Carlos; Antonio Navarro, Rondán, Francisco Javier y Rodríguez, Carlos Javier (2008), Unidades franquiciadas versus propias en el sistema franquiciador: Una investigación empírica, Investigaciones Europeas de Dirección y Economía de la Empresa, Vol. 14, $\mathrm{N}^{\circ}$ 2, Academia Europea de Dirección y Economía de la Empresa (AEDEM virtual), pp 185210.

Duarte, Paulo Alexandre y Raposo Mario Lino (2010), A PLS Model to Study Brand Preference: An Application to the Mobile Phone Market. Esposito
Vinzi y otros. (eds.) Handbook of Partial Least Squares, Springer Handbooks of Computational Statistics: Heidelberg.

Fassott, Georg (2006), Operationalisierung latenter Variablen in Strukturgleichungsmodellen: Eine Standortbestimmung,

Schmalenbachs Zeitschrift Für Betriebswirtschaftliche Forschung. Editorial Springer, Vol. 58, 67-88.

Fornell, Claes y Larcker, David (1981), Evaluating structural equation models with unobservable variables and measurement error, Journal of Marketing Research, Vol.18, $\mathrm{N}^{\circ} .1$, Macquarie University Press, 03090566, pp 39-50.

Gillis, William y Castrogiovanni, Gary (2012), The franchising business model: an entrepreneurial growth alternative, International Entrepreneurship and Management Journal, Vol. 8, $\mathrm{N}^{\circ}$. 1 , Editorial Springer Science+Business Media, pp 75-98.

Grünhagen, Marko y Mittelstaedt, Robert (2005), Entrepreneurs or Investors: Do Multi-unit Franchisees Have Different Philosophical Orientations?, Journal of Small Business Management, Vol. 43, $\mathrm{N}^{\circ} .3$, Universidad de Nebraska-Lincon, Alemania, pp 207-225

Hair, Joseph; Babin, Barry; Black, William; Anderson, Rolf y Tatham, R. L. (2006), Multivariate data analysis. New Jersey: (6th ed.) Pearson Education International.

Hair, Joe; Ringle, Christian y Sarstedt, Marko (2011), PLS-SEM: Indeed a Silver Bulleten. Journal of Marketing Theory and Practice,Vol. 19, No. 2, Editorial Taylor \& Francis Online, $\mathrm{pp}$ 139-152.

Henseler, JÖrg; Ringle, Christian y Sinkovics, Rudolf (2008), The Use of Partia Least Squares Path Modeling in International Marketing, Advances in International Marketing, $\mathrm{N}^{\circ}$. 19, Vol. 20, Social Science Resarch Network, pp 277-320.

Ho, Y.F. y Wang, H.L. (2008), Applying fuzzy Delphi method to select the 
variables of a sustainable urban system dynamics model, the 26th International Conference of system Dynamics Society, University of Patras, Greece. Disponible en: http://www.systemdynamics.org/ conferences/2008/proceed/papers/ HO311.pdf

Hoffman, Richard y Preble, John (2003), Convert to compete: Competitive advantage through conversion franchising, Journal of Small Business Management, Vol. 41, No. 2, Editorial Wiley Online Library, pp 187-204. DOI:10.1111/1540$627 \times .00075$

Hulland, John (1999), Use of partial least squares (PLS) in strategic management research: a review of four recent studies, Strategic Management Journal, No. 20, Editorial Wiley Online Library, pp 195-204. DOI: 10.1002/(SICl)1097$0266(199902) 20: 2<195::$ AID SMJ13>3.0.CO;2-7.

Instituto Nacional de Estadística, Geografía e Informática INEGI (2012), Catálogo Nacional de Franquicias. México. Disponible en: http://www.inegi.org. $\mathrm{mx} /$ prod_serv/contenidos/espanol/ bvinegi/productos/metodologias/ ENOE/ENOE2012/franquicias/ franquicias_2012.pdf. Fecha de consulta 30 de junio de 2014

Jacob, Enrique (2014), Conferencia inaugural en la Feria Internacional de Franquicias. Asociación Mexicana de Franquicias. México, D. F.

Lafontaine, Francine y Oxley, Joanne (2004), International franchising practices in Mexico: Do franchisors customize their contracts? Journal of Economics y Management Strategy, Vol. 13, $\mathrm{N}^{\circ}$. 1, Editorial Wiley Online Library, $\mathrm{pp}$ 95-123.

Lillis, Charles, Chem. Narayama y Gilman, John (1976), Competitive advantage variation over the life cycle of a franchiseen, The Journal of Marketing, Vol. 40, $\mathrm{N}^{\circ}$. 4, Editorial American Marketing Association Journals, pp 77-80.

Niefert, Michaela (2010), Characteristics and Determinants of Start-ups from
Unemployment: Evidence from German Micro Data, Journal of Small Business and Entrepreneurship, $\mathrm{N}^{\circ}$. 23. Vol. 23, Editorial Taylor Francis Online, pp 409-429.

Norton, William y Moore, William (2006), The Influence of Entrepreneurial Risk Assessment on Venture Launch or Growth Decisions, Small Business Economics, $\mathrm{N}^{\circ}$. 3, Vol. 26, Editorial Springer, pp 215-226.

Penrose, Edith (1962), Teoría del Crecimiento de la Empresa. Ed. Aguilar, S. A. Madrid, Traducción del libro The theory of the Growth of the Firm, Oxford: John Wiley y Sons, 1959. Disponible en: http://www.worldcat.org/title/teoriadel-crecimiento-de-la-empresa/ $\mathrm{clc} / 803073757$ ? referer $=$ di\&ht=edition . Fecha de consulta: 23 de diciembre de 2008.

Petter, Stacie, Straub, Detmar, y Rai, Arun (2007), Specifying Formative Constructs in Information Systems Research, Vol. 31, N.4, Editorial MS Quarterly, pp 623-656.

Peteraf, Margaret A. (1993), The cornerstones of competitive advantage. A resourcebased View, Strategic management Journal, Vol.14, $\mathrm{N}^{\circ} .3$, Editorial MIS Quarterly, pp 179-191.

Pilling, Bruce (1991), Assessing competitive advantage in small business: An application to franchising, Journal of Small Business Management, Vol. 29, N. 4, Editorial ProQuest, pp 5583.

Porter, Michael (1982), Estrategia competitiva. Técnicas para el Análisis de los Sectores Industriales de la Competencia. Editorial CECSA. Editorial 32. México. 289.

Porter, Michael (1985). Competitive advantage. The Free Pres, Nueva York. Harvard Business School, Estados Unidos.

Porter, Michael (1990), The Competitive Advantage of Nations, Free Press New York. Disponible en: http:// kkozak.wz.cz/Porter.pdf Fecha de consulta: 4 de enero de 2006.

Ramírez, José Manuel; Rondán, Francisco Javiery Guerrero, Flor María (2007), 
Selección de franquiciados mediante simulación con análisis conjunto, Cuadernos de Economía y Dirección de la Empresa, Vol. 10, N. 31, Editorial Elsevier, pp 43-62.

Revuelto-Taboada, Lorenzo; Saorín-lborra, M. Carmen y Fernández-Guerrero, Rafael (2011), Hacia una perspectiva integradora de la teoría de grupos estratégicos: validez convergente $y$ fortaleza de la definición de grupo estratégico, INNOVAR, Vol. 21, N. 40, Bogotá, Colombia, pp 199-218.

Reynolds, Paul D.; Camp, S. Michael; Bygrave, William D.; Autio, Erkko. y Hay, Michael (2001), Global Entrepreneurship Monitor, 2002. Executive Report Babson. College. London Business School and Kaufman Center for Entrepreneurial Leadership.

Ringle, Christian; Sven, Wende y Will, Alexander (2005), Smartpls 2.0 (Beta). Hamburg, Germany: University of Hamburg. Disponible en http://www.smartpls.de. Fecha de consulta: 11 de octubre de 2014.

Saavedra. María Luisa (2012), Hacia la competitividad de la pyme latinoamericana. Macroproyecto de investigación. Asamblea ALAFEC, Buenos Aires, Argentina. Disponible en: http://www.alafec.unam.mx/docs/ macroproyectos/competitividad_macro. pdf Fecha de consulta: 27 de octubre de 2012.

Sardy, Marc y Alon, Ilan (2007), Exploring the differences between franchisee entrepreneurs and nascent entrepreneurs, International Entrepreneurship and Management Journal, Vol. 3, No. 4, Editorial Springer, pp 403-418.

Schumpeter, Joseph (1934), A. The Theory of Economic Development. Cambridge, Mass. Harvard University Press. Harvard Economics Studies 46, Estados Unidos, pp 255. Disponible en: <http://books.google.es/books> Fecha de consulta: 9 de septiembre de 2005.

Seidel, Gunter y Back, Andrea (2009), Success factor validation for global en ERP $17^{\text {th }}$. European Conference on Information Systems. Disponible en: http://intranet.iwi.unisg.ch/org/ iwi/iwi_pub.nsf/wwwAuthorPublGer/ F9D89C12577FC003A7AF2/\$file/ ecis2009-0098.pdf Fecha de consulta: 5 de enero de 2012.

Sekaran, Uma (2002), Research Methods for Business: A Skill-Building Approach, 4 ${ }^{\text {rd }}$ Edición, Wiley: New York, USA. Disponible en: http:// www.wiley.com/WileyCDA/WileyTitle/ productCd-0471203661.html Fecha de consulta: 2 de marzo de 2006.

Shane, Scott; Shankar, Venkatesh; Aravindakshan, Ashwin (2006), The Effects of New Franchisor Partnering Strategies on Franchise System Size, Management Science, Vol. 52 , No. 5, ResearchGate Publications INFORMS, pp 773 - 787.

Tormo y Asociados (2012), La franquicia en el mundo. Informes y Publicaciones. Disponible en: http://www.tormoasociados.es/Informes/informes.aspx Fecha de consulta: 7 de Julio de 2013.

Tuunanen, Mika y Hyrsky, Kimmo (2001), Entrepreneurial Paradoxes in Business Format Franchising: An Empirical Survey of Finnish Franchisees, International Small Business Journal, Vol. 19, No. 47, Editorial SAGE Publications, pp 4762.

Urbano, David y Toledano, Nuria (2008), Invitación al emprendimiento: una aproximación a la creación de empresas. Barcelona: UOC. Disponible en: http:// www.davidurbano.es/pdf/Libros/ InvitacionAIEmprendimiento_2008. pdf Fecha de consulta: 22 de julio de 2009.

Verheul, Ingrid; van Stel, André; Thurik, Roy y Urbano, David (2006), The Relationship between Business Ownership and Unemployment in Spain: A Matter of Quantity or Quality?, Estudios de Economía Aplicada, Vol. 24, No. 2, España, pp 435-457.

Welsh, Dianne; Alon, Ilan y Falbe, Cecilia (2006), An Examination of 
International Retail Franchising in Emerging Markets, Journal of Small Business Management, Vol. 44, No. 1, Wiley OnLine Library, pp 130-149.

Wennekers, Sander y Thurik, Roy (1999), Linking Entrepreneurship and Economic Growth, Small Business Economics, No. 1, Vol. 13, Editorial Springer, pp 27-56.

Wernerfelt, Birger. (1984), A resourcebased view of the firm. Strategic Management Journal, Vol. 5, No. 2, Universidad de Michigan, USA.
Editorial John Wiley \& Sons, pp 171-180.

Wetzels, Martin; Odekerken-Schroder, Gaby; and van Oppen, Claudia (2009), Using PLS path modelling for assessing hierarchical construct models: guidelines and empirical illustration, MIS Quarterly, Vol. 33, No. 1 Management Information Systems Research Center, University of Minnesota, pp 177-195. 